\title{
Sobre a ocorrência da moléstia de Chagas no Estado do Amazonas, Brasil ( $\left.{ }^{*}\right)$
}

\author{
Flávio Barbosa de Almeida $(* *)$
}

J. A. Nunes de Mello ( $\left.{ }^{(*)}\right)$

\section{Resumo}

Em área do Estado do Amazonas (Município de Barcelos) onde recentemente foram assinalados seis casos humanos suspeitos de moléstia de Chagas por sorologia positiva, procuramos o Trypanosoma cruzi em 35 pessoas, 9 cães domésticos e 71 mamíferos silvestres. Todos foram negativos para tripanosomas, exceto 6 morcegos de quatro espécies, que albergavam flagelados com caracteristicas do subgênero Schizotrypanum: Carollia perspicilata, Glossophaga soricina, Desmodus rotundus e Mollossidae sp. Cultura do flagelado de um dos $\mathbf{G}$. soricina infectou camundongo, caráter que o aproxima do T. cruzi. Todos os triatomineos que capturamos - Panstrongylus rufotuberculatus, Eratyrus mucronatus, Rhodnius pictipes e R. brethesi - foram negativos para flagelados.

\section{INTRODUÇÃo}

A presença do Trypanosoma cruzi no Estado do Amazonas em hospedeiros vertebrados ou invertebrados já foi observada por diversos pesquisadores (Almeida, 1971; Almeida \& Deane, 1970; Almeida \& Machado, 1971; Almeida \& Santos, 1975; Almeida et al, 1971 e Deane et al, 1972). Contudo, nenhum caso humano foi ainda descrito, embora Ferraroni et al. (1977) houvessem achado seis casos suspeitos sorologicamente positivos, em área do Município de Barcelos, Estado do Amazonas.

Em 1977, por duas vezes estivemos nos rios Aracá, Demeni e Padauari, tributários do rio Negro, Barcelos (Mapa), no período de 14.07.77 a 24.08 .77 e de 19.10 .77 a 09.11 .77 . interessados no estudo da biologia dos triatomíneos silvestres encontradiços em palmeiras da região. Esse município é importante pelo seu comércio extrativista de fibras vegetais procedentes de palmeiras que constituem habitat natural de triatomíneos. Sua sede é ponto de confluência daqueles produtos regionais e está situada à margem direita do rio Negro, tendo as seguintes coordenadas geográficas: $0^{\circ} 58^{\prime} 01^{\prime \prime}$ - latitude Sul, $62^{\circ} 56^{\prime} 00^{\prime \prime}$ longitude W. Gr.; fica a $403 \mathrm{~km}$ a NO de Manaus, em linha reta.

\section{MATERIAL E MÉTODOS}

Por punção venosa foi colhido sangue de 32 pessoas, adultas, todas moradoras do rio Padauari, o mais populoso dos três visitados. Desse material foram feitos: esfregaço e gota espessa corados pelo Giemsa, inoculação em ratos e camundongos por via intraperitoneal e semeadura em meio de cultura líquido, segundo Warren, ligeiramente modificado.

Realizamos xenodiagnóstico em seis pessoas, de três das quais não pudemos colher sangue. Para cada pessoa utilizamos dez ninfas de $5.0^{\circ}$ estádio de Rhodnius neglectus (gentileza do Prof. Humberto Menezes da Faculda. de de Medicina de Ribeirão Preto, SP) e uma de Rhodnius brethesi, criada de material da própria localidade-tipo, o rio Aracá, Barcelos, AM. A exiguidade de material disponível na ocasião limitou o número de xenodiagnósticos. $\mathrm{Na}$ oportunidade trabalhamos ainda com cães domésticos que eram sangrados e libertados.

Para a identificação de possíveis reservatórios silvestres de Trypanosoma cruzi, os mamíferos silvestres foram abatidos a tiro e imediatamente sangrados; os morcegos foram capturados com redes especiais e sangrados posteriormente na lancha-laboratório.

Os mamíferos silvestres foram identificados quanto ao seu nome vulgar e científico, conservando-se a pele e o crânio quando existia dúvida. Os quirópteros sofreram idêntico tratamento, mas pela complexidade de sua identificação e tamanho diminuto, foram conservados imersos em formol a $2 \%$. O sangue

(*) - Financiado pelo Conselho Nacional de Desenvolvimento Científico e Tecnológico (CNPq).

(**) - Instituto Nacional de Pesquisas da Amazônia, Manaus. 
dos animais sofreu o mesmo tratamento referido para os casos humanos, porém dos animais conservamos também pedaços de vísceras (fígado, baço e coração) em formol a $10 \%$, para estudos posteriores.

A captura de triatomíneos foi feita pelo abate de palmeiras e procura dos insetos entre as fibras e folhas; os hemípteros foram conservados vivos para criação em laboratório, após coleta de fezes, por compressão abdominal.

\section{RESUltados}

As 35 pessoas examinadas, inclusive 4 das 6 sorologicamente positivas (Tabela I), bem como os nove cães domésticos, foram todos negativos para Trypanosoma cruzi.

Dos 71 mamíferos silvestres examinados (Tabela II), 6 morcegos albergavam tripanosomas com aspecto característico do subgênero Schizotrypanum; em um Carollia perspicillata e um Glossophaga soricina os flagelados eram morfologicamente indistinguíveis do Trypanosoma cruzi e hemocultura, do G. soricina ricas em tripomastigotas metacíclicos infectaram camundongo inoculado intraperitonealmente. Em um C. perspicillata, um G. soricina, um Desmodus rotundus e um Mollossidae sp. os tripanosomas tinham o aspecto atribuído ao $T$. vespertilionis, por seu tamanho pequeno e índice nuclear alto, mas não se desenvolveram em meio de cultura.

Em um Didelphis marsupialis (mucura) foi observado o Trypanosoma freitasi.

Em três macacos Cacajao melanocephalus foi achado um tripanosoma semelhante ao $T$. mycetae.

Uma paca, Cuniculus paca, albergava um Trypanosoma sp. que está sendo estudado.

Duas espécies de triatomíneos foram capturadas em nossa lancha-laboratório, o Panstrongylus rufotuherculatus e o Eratyrus mucro-

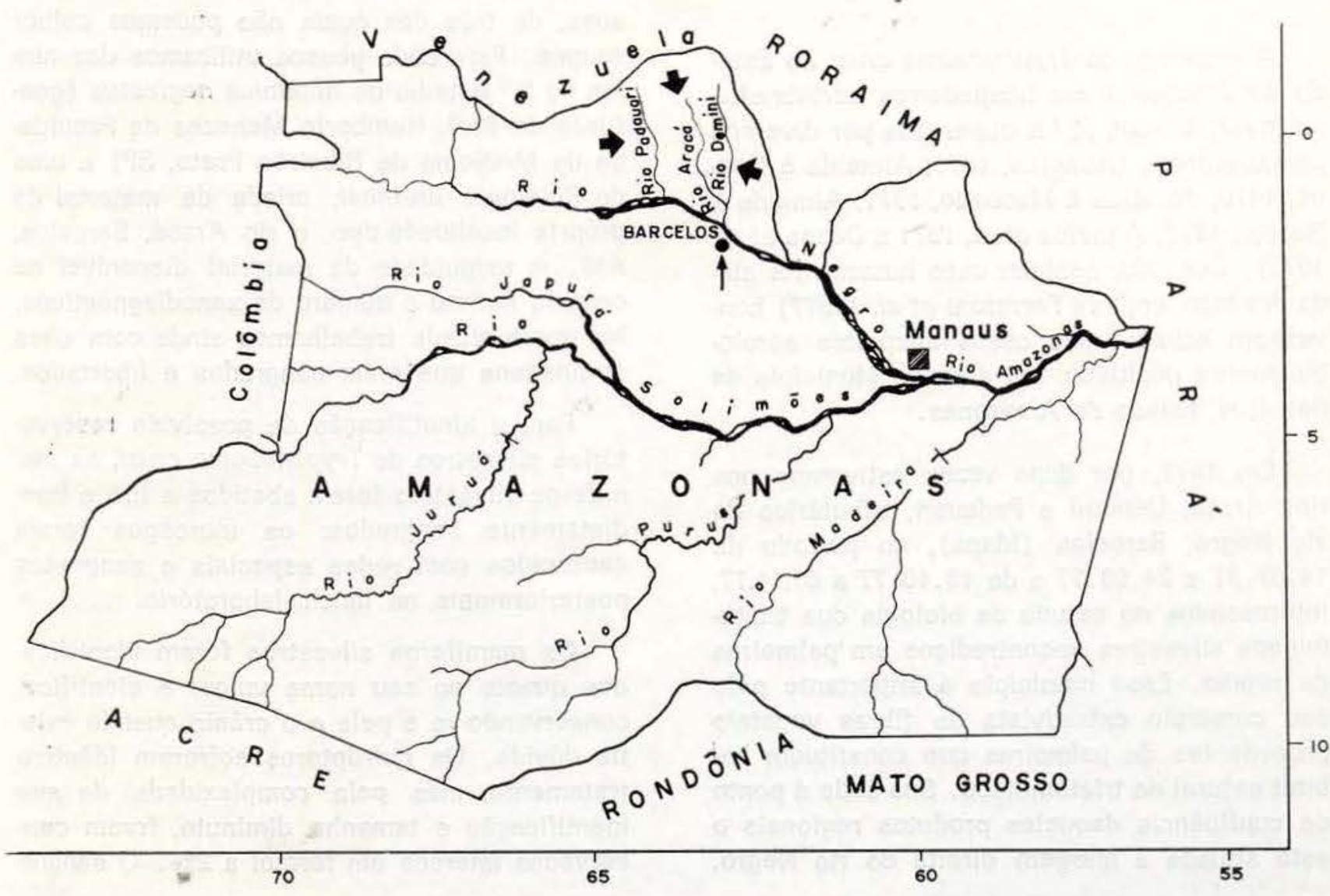

Localização geográfica dos rios Demeni, Aracá e Padauari, no Município de Barcelos, Estado do Amazonas, Brasil 
natus, provavelmente atraídos pela iluminação por lâmpadas fluorescentes. Ninfas e adultos de Rhodnius pictipes foram coletados em palmeira inajá, Maximiliana martiana, no rio Demeni. No rio Aracá foi coletada somente a espécie típica do local, Rhodnius brethesi (Tabela III). Todos os triatomíneos mostraram-se negativos para flagelados.

TABELA I - Resultado do exame das 35 pessoas com vistas ao T. cruzi, no Município de Barcelos, Estado do Amazonas, Brasil

\begin{tabular}{|c|c|c|c|c|c|c|}
\hline Ident. & Idode & Sexo & $\begin{array}{l}\text { Sangue: } \\
\text { gota os- } \\
\text { pessa e } \\
\text { esfragaço }\end{array}$ & $\begin{array}{l}\text { Inoculaçảo } \\
\text { de sangue } \\
\text { em rato } \\
\text { e camun- } \\
\text { dongo }\end{array}$ & $\begin{array}{l}\text { Xeno- } \\
\text { diagnós- } \\
\text { tico }\end{array}$ & $\begin{array}{l}\text { Hemo- } \\
\text { cultu- } \\
\text { ra }\end{array}$ \\
\hline
\end{tabular}

\begin{tabular}{lcccccc}
\hline AB & 49 & M & - & $\ldots$ & $\ldots$ & - \\
TJB & 60 & F & - & $\ldots$ & $\ldots$ & - \\
LL. & 35 & M & - & - & - & - \\
AOS & 54 & F & - & $\ldots$ & $\ldots$ & - \\
JTS & 58 & M & - & $\ldots$ & $\ldots$ & - \\
ROM & 18 & M & - & $\ldots$ & $\ldots$ & - \\
ES & 25 & F & - & $\ldots$ & $\ldots$ & - \\
EOS & 28 & M & - & $\ldots$ & $\ldots$ & - \\
SOS & 19 & M & - & $\ldots$ & $\ldots$ & - \\
JOM & 21 & M & - & $\ldots$ & $\ldots$ & - \\
JBG & 15 & F & - & $\ldots$ & $\ldots$ & - \\
JAP & 48 & M & - & $\ldots$ & $\ldots$ & - \\
MCZ & 13 & F & - & $\ldots$ & $\ldots$ & - \\
JN & 48 & F & - & $\ldots$ & $\ldots$ & - \\
NB & 45 & F & - & $\ldots$ & $\ldots$ & - \\
RBL & 30 & F & - & $\ldots$ & $\ldots$ & - \\
JG & 67 & M & - & $\ldots$ & $\ldots$ & - \\
DG & 27 & F & - & $\ldots$ & $\ldots$ & - \\
FPM & 25 & M & - & $\ldots$ & $\ldots$ & - \\
MAS & 19 & F & - & $\ldots$ & $\ldots$ & - \\
CO & 48 & M & - & $\ldots$ & - & - \\
WPS & 27 & M & - & $\ldots$ & $\ldots$ & - \\
DM & 24 & F & - & $\ldots$ & $\ldots$ & - \\
MO & 50 & F & - & - & - & - \\
MASS & 23 & F & - & $\ldots$ & $\ldots$ & - \\
AASF & 23 & M & - & $\ldots$ & $\ldots$ & - \\
AAS & 28 & M & - & $\ldots$ & $\ldots$ & - \\
AAP & 57 & M & - & - & $\ldots$ & - \\
FAP & 20 & M & - & $\ldots$ & $\ldots$ & - \\
CAC & 41 & M & - & $\ldots$ & $\ldots$ & - \\
AVS & 18 & M & - & $\ldots$ & $\ldots$ & - \\
FF & 48 & M & - & $\ldots$ & $\ldots$ & - \\
JCO & 43 & M & $\ldots$ & $\ldots$ & - & $\ldots$ \\
JAU & 41 & M & $\ldots$ & $\ldots$ & - & $\ldots$ \\
NB & 49 & F & $\ldots$ & $\ldots$ & - & $\ldots$ \\
& & & & & & \\
\hline & & & & & \\
\hline
\end{tabular}

(*) Com seis reaçóes sorológicas positivas (Ferraroni et al., 1977).

\section{Discussão}

O fato de não termos evidenciado o agente etiológico da moléstia de Chagas em Barcelos, $\mathrm{AM}$, não nos autoriza negar a ocorrência daquela parasitose na área, isto porque o número de pessoas examinadas foi pequeno e notadamente reduzido o número de xenodiagnósticos.

TABELA II - Exame de sangue de mamíferos silvestres do Município de Barcelos, Estado do Amazonas, Brasil

\begin{tabular}{|c|c|c|c|}
\hline Espécie de momífero & $\begin{array}{l}\text { Exami- } \\
\text { nados }\end{array}$ & $\begin{array}{l}\text { Com } \\
\text { Trypano- } \\
\text { soma } \\
\text { (Schizotri- } \\
\text { panum) } \\
\text { sp. }\end{array}$ & $\begin{array}{l}\text { Outros } \\
\text { Tripa- } \\
\text { noso- } \\
\text { mas }\end{array}$ \\
\hline CHIROPTERA & & & \\
\hline Desmodus rotundus ........ & 10 & 1 & - \\
\hline Ectophylla macconelli ..... & 15 & - & - \\
\hline Glossophaga soricina ...... & 6 & $2^{*}$ & - \\
\hline Carollia perspicillata...$\ldots$ & 5 & 2 & - \\
\hline Artibeus lituratus $\ldots \ldots \ldots \ldots$ & 4 & - & - \\
\hline Artibeus cinereus $\ldots \ldots \ldots$ & 3 & - & 一 \\
\hline $\begin{array}{c}\text { Mollossidae sp. } \ldots \ldots \ldots \ldots \\
\text { RODENTIA }\end{array}$ & 2 & 1 & 一 \\
\hline Cuniculus paca $\ldots \ldots \ldots \ldots$ & 5 & - & 1 \\
\hline Echimydae sp. ........... & 5 & - & 一 \\
\hline Oryzomys sp. $\ldots \ldots \ldots \ldots$ & 1 & - & 一 \\
\hline $\begin{array}{l}\text { Sciurus igniventris } \ldots \ldots \ldots \\
\text { PRIMATES }\end{array}$ & 1 & - & - \\
\hline Alouatta seniculus straminea & 4 & 一 & 一 \\
\hline $\begin{array}{c}\text { Cacajao melanocephalus ... } \\
\text { MARSUPIALIA }\end{array}$ & 4 & 一 & 3 \\
\hline $\begin{array}{c}\text { Didelphis marsupialis } \ldots \ldots \\
\text { CARNIVORA }\end{array}$ & 2 & - & 1 \\
\hline Potos flavus $\ldots \ldots \ldots \ldots \ldots$ & 1 & - & - \\
\hline $\begin{array}{c}\text { Bassaricyon gabbii } \ldots \ldots \ldots \ldots \\
\text { ARTIODACTYLA }\end{array}$ & 1 & - & - \\
\hline Tayassu pecari.$\ldots \ldots \ldots \ldots$ & 1 & - & - \\
\hline Tayassu tacaju $\ldots \ldots \ldots \ldots$ & 1 & 一 & - \\
\hline TOTAL $\ldots . . . .$. & 71 & 6 & 5 \\
\hline
\end{tabular}

(*) Hemoculturas de um deles infectaram camundongo, sendo provavelmente $\mathbf{T}$. cruxi. 
TABELA III - Triatomíneos capturados no Município de Barcelos, Estado do Amazonas, Brasil

\begin{tabular}{|c|c|c|}
\hline Espécie & $\begin{array}{c}\text { Rio } \\
\text { Demeni }\end{array}$ & $\begin{array}{c}\text { Rio } \\
\text { Aracá }\end{array}$ \\
\hline Panstrongylus rufotuberculatus " . & 2 & - \\
\hline Eratyrus mucronatus ${ }^{*} \ldots \ldots \ldots$ & 2 & - \\
\hline Rhodnius brethesi * $\ldots \ldots \ldots \ldots$ & - & 39 \\
\hline Rhodnius pictipes $* \ldots \ldots \ldots \ldots$ & 5 & - \\
\hline$\ldots \ldots \ldots \ldots \ldots$ & 9 & 39 \\
\hline
\end{tabular}

(*) Capturado no barco, à noite.

(*) Capturado em palmeiras (piaçaba e inajá).

Quanto aos tripanosomas com aspecto de Schizotrypanum encontrados nos morcegos, não podemos chegar a um diagnóstico específico definitivo. As formas morfologicamente indistinguiveis do $T$. cruzi que achamos em um Carollia perspicillata, um Glossophaga soricina cultivam bem e hemoculturas do G. soricina infectaram camundongo; é provável que esta última cepa seja de $T$. cruzi, embora não tenhamos outros dados (p. ex., xenodiagnóstico) habitualmente utilizados na identificação. Os flagelados com aspecto de $T$. vespertilionis achados em outros morcegos também não podem ser confirmados nessa espécie: $T$. vespertilionis, ao contrário do nosso material, se desenvolve bem em meios de cultura e não evolui em triatomíneos, caráter que não investigamos, pois não fizemos xenodiagnósticos.

Os Schizotrypanum dos morcegos (rio Demeni), não foram observados propriamente no local dos suspeitos do Mal de Chagas (rio Padauari), porém é de se estranhar que, naquele local, não encontrassemos nenhum mamífero silvestre, cão doméstico ou triatomíneos com $T$. cruzi. Rodrigues \& Melo (1942) em Aurá no Estado do Pará, encontraram em maníferos silvestres e num cão doméstico e ainda em dưas espécies de triatomíneos em local onde todos os exames feitos em indivíduos humanos foram negativos. Matta (1919) nenhuma referência fez sobre a ocorrência do T. cruzi em Barcelos,. Estado do Amazonas, quando descreveu o Rhodnius brethesi do rio Aracá. Em alguns municípios do Estado do Amazonas não é raro o encontro de tripanosomas com aspecto do $T$. cruzi em Didelphis (mucuras), Saimiri sciureus (macacos) e Quirópteros (morcegos) e em quase todas as espécies de triatomíneos de Manaus.

\section{AGRADECIMENTOS}

Os AA. manifestam, aqui, seus agradecimentos ao Prof. Leonidas Mello Deane (Faculdad de Medicina, Universidad de Carabobo, Valencia, Venezuela), pelas sugestöes apresentadas na apreciação do manuscrito; ao Prof. Humberto Menezes (Faculdade de Medicina de Ribeirão Preto, SP) pelas ninfas dos triatomíneos utilizadas neste trabalho.

\section{SUMMARY}

In the municipality of Barcelos, State of Amazonas, Brazil, where six humans have been recently reported with positive serological tests por Chagas' disease, we have searched Trypanosoma cruzi in 35 persons, 9 dogs and 71 wild mammals. All were negative for hemoflagellates, except six bats of 'four species, which harboured trypanosomes of the subgenus Schizotrypanum : Carollia perspicillata, Glossophaga soricina, Desmodus rotundus and Mollossidae sp. A strain from one of the $\mathbf{G}$. soricina is probably $\mathbf{T}$. cruzi, since hemocultures infected a laboratory mouse. All triatomine bugs captured in the area - Panstrongylus rufotuberculatus, Eratyrus mucronatus, Rhodnius pictipes and R. brethesi - were free of flagellates.

\section{BIBLIOGRAFIA CITADA}

Almeida, F. B.

1971 - Triatomíneos da Amazônia. Encontro de três espécies naturalmente infectadas por Trypanosoma semelhante ao cruzi no Estado do Amazonas (Hemiptera, Reduviidae). Acta Amazonica, 1(1): 89-93.

Almeida, F. B. \& DeAne, L. M.

1970 - Plasmodium brasilianum reencontrado em seu hospedeiro original, o macaco uacari branco, Cacajao calvus. Bol. INPA (Patologia Tropical), Manaus, $4: 1-9$.

ALMeIdA, F. B. \& MACHAdo, P. A.

1971 - Sôbre a infecção natural do Panstrongylus geniculatus pelo Trypanosoma cruzi em Manaus, Amazonas, Brasil. (Apresentado no 
VII Congresso da Sociedade Brasileira de Medicina Tropical, Manaus, AM). Acta Amazonica, 1(2): $71-75$.

Almeida, F. B. \& Santso, E. I.

1975 - Tripanosomas encontrados em quirópteros de Codajás, Estado do Amazonas, Brasil. (Apresentado no XI Congresso da SBMT, Rio de Janeiro, RJ).

Almeida, F. B.; Albuquerque, L. P. \& Machado, P. A.

1971 - Vetores e reservatórios silvestres de tripanosoma na região de Manaus. (Apresentado no VII Congresso da SBMT, Manaus, AM).

Deane, L. M.; Almetda, F. B.; Ferretra Neto, J. A. \& Silva, J. E.
1972 - Trypanosoma cruzi e outros tripanosomas em primatas brasileiros (Apresentado no VIII Congresso da SBMT, B. Horizonte, MG).

Ferraroni, J. J.; Nunes de Mello, J. A. \& Camargo, M. E.

1977 - Moléstia de Chagas na Amazônia. Ocorrência de seis casos suspeitos, autóctones sorologicamente positivos. Acta Amazonica, $7(3): 438-440$.

Matta, A.

1919 - Um novo Redúvido do Amazonas. Rhodnius Brethesi N. Sp. Amazonas Medico, 2(3) : 93-94.

Rodrigues, B. A. \& Melo, G. B.

1942 - Contribuição ao Estudo da Tripanosomiase Americana. Mem. Inst. Oswaldo Cruz, $37: 41-90$.

(Aceito para publicação em $08 / 11 / 78$ ) 